\title{
Potential and Kinetic Shaping for Control of Underactuated Mechanical Systems
}

\author{
Anthony M. Bloch \\ Department of Mathematics \\ University of Michigan \\ Ann Arbor, MI 48109 \\ email: abloch@umich.edu \\ Naomi Ehrich Leonard \\ Mechanical and Aerospace Eng. \\ Princeton University \\ Princeton, NJ 08544 \\ email: naomi@princeton.edu
}

Proc. Amer. Control Conf., (2000), 3913-3917.

\begin{abstract}
This paper combines techniques of potential shaping with those of kinetic shaping to produce some new methods for stabilization of mechanical control systems. As with each of the techniques themselves, our method employs energy methods and the LaSalle invariance principle. We give explicit criteria for asymptotic stabilization of equilibria of mechanical systems which, in the absence of controls, have a kinetic energy function that is invariant under an Abelian group.
\end{abstract}

\section{Introduction}

The method of controlled Lagrangians is a constructive approach to the derivation of stabilizing control laws for underactuated Lagrangian mechanical systems with symmetry and with broken symmetry (Bloch, Leonard and Marsden [1997,1998,1999a,1999b,1999c], Bloch, Chang, Leonard and Marsden [1999], Bloch, Chang, Leonard, Marsden and Woolsey [2000]). As part of this method, we consider control laws that yield closed-loop dynamics in Lagrangian form so that stabilization can be understood in terms of energy shaping. Other relevant work involving energy methods in control and stabilization includes Wang and Krishnaprasad [1992], Koditschek and Rimon [1990], Baillieul [1993], Spong [1996], Åström and Furuta [1996], Leonard [1997], Auckly, Kapitanski and White [1998] and Hamberg [1999].

This paper combines methods for control of underactuated systems that involve Lie bracket computations (Poisson brackets in the case of Hamiltonian systems) with the controlled Lagrangian technique. In particu-

\author{
Dong Eui Chang \\ Control and Dynamical Systems 107-81 \\ California Institute of Technology \\ Pasadena, CA 91125 \\ email: dchang@cds.caltech.edu \\ Jerrold E. Marsden \\ Control and Dynamical Systems 107-81 \\ California Institute of Technology \\ Pasadena, CA 91125 \\ email: marsden@cds.caltech.edu
}

lar, we explore how potential shaping in the sense of van der Schaft [1986] and Jalnapurkar and Marsden $[1999,2000]$ can be combined with kinetic shaping using controlled Lagrangians.

Assume the given mechanical system has configuration space $Q$ and that a Lie group $G$ acts freely and properly on $Q$. An important special case is that where $Q=S \times G$ with $G$ acting only on the second factor by left group multiplication. For example, for the inverted planar pendulum on a cart, $Q=S^{1} \times \mathbb{R}$ with $G=\mathbb{R}$, the group of reals under addition (corresponding to translations of the cart). We are interested in the underactuated problem in which the controls act directly only on the variables lying in $G$, but all variables in the state space are to be controlled. We suppose that $G$ is a symmetry group for the kinetic energy of the system but the potential energy $V$ need not be $G$ invariant.

Let $\theta^{a}$ be coordinates for $G$ and $x^{\alpha}$ be coordinates for $Q / G$. Let the metric tensor $g(\cdot, \cdot)$ define the kinetic energy $\frac{1}{2} g(\dot{q}, \dot{q})$ and let $L: T Q \rightarrow \mathbb{R}$ be the original Lagrangian given by the kinetic minus potential energy:

$$
\begin{aligned}
L\left(x^{\alpha}, \theta^{a}, \dot{x}^{\alpha}, \dot{\theta}^{a}\right) & =\frac{1}{2} g_{\alpha \beta} \dot{x}^{\alpha} \dot{x}^{\beta}+g_{\alpha a} \dot{x}^{\alpha} \dot{\theta}^{a}+\frac{1}{2} g_{a b} \dot{\theta}^{a} \dot{\theta}^{b} \\
& -V\left(x^{\alpha}, \theta^{a}\right) ;
\end{aligned}
$$

$\left(x_{e}, \theta_{e}, 0,0\right) \in T Q$ is the equilibrium of interest where $\left(x_{e}, \theta_{e}\right)$ is a critical point of the original potential $V$.

\section{Potential Shaping.}

We first review some of the techniques of potential shaping. To illustrate these, we consider a case where potential shaping alone is sufficient to achieve asymptotic stabilization. 
Assume the following definiteness condition:

$$
\frac{\partial^{2} V}{\partial x^{\alpha} \partial x^{\beta}}\left(x_{e}, \theta_{e}\right)>0,
$$

i.e., the equilibrium is a minimum of the original potential energy in the $x^{\alpha}$ variables. Notice that this excludes the examples treated in the method of controlled Lagrangians where the equilibrium is a maximum of the given potential energy. If (2.1) holds, the Lyapunov stabilization of the equilibrium $\left(x_{e}, \theta_{e}, 0,0\right)$ can be achieved by potential shaping. Indeed, choose any function $V_{\epsilon}: G \rightarrow \mathbb{R}$ with a minimum at $\theta_{e}$ and let the control input $u$ be of the form

$$
u_{a}=-\frac{\partial V_{\epsilon}}{\partial \theta^{a}}+\tilde{u}_{a}
$$

Then, we can check that the Euler-Lagrange equations of the given Lagrangian $L$ with the force $u$ are equal to those of the new Lagrangian $\tilde{L}$ defined by

$$
\begin{aligned}
\tilde{L}\left(x^{\alpha}, \theta, \dot{x}^{\alpha}, \dot{\theta}\right) & =L\left(x^{\alpha}, \theta, \dot{x}^{\alpha}, \dot{\theta}\right)-V_{\epsilon}\left(\theta^{a}\right) \\
& =K\left(x^{\alpha}, \theta^{a}, \dot{x}^{\alpha}, \dot{\theta}^{a}\right)-\tilde{V}\left(x^{\alpha}, \theta^{a}\right)
\end{aligned}
$$

with the force $\tilde{u}$ where $\tilde{V}=V+V_{\epsilon}$. Let $\tilde{E}$ be the energy from the Lagrangian $\tilde{L}$ defined by $\tilde{E}=K+\tilde{V}$. By the choice of $V_{\epsilon},\left(x_{e}, \theta_{e}, 0,0\right)$ is a critical point of $\tilde{E}$. The second derivative of $\tilde{E}$ at $z_{e}=\left(x_{e}, \theta_{e}, 0,0\right)$ is

$$
D^{2} \tilde{E}\left(z_{e}\right)=\left.\left[\begin{array}{cc}
D^{2} \tilde{V} & 0 \\
0 & D^{2} K
\end{array}\right]\right|_{z=z_{e}}
$$

where $D^{2} \tilde{V}$ is given by

$$
D^{2} \tilde{V}=\left[\begin{array}{cc}
\frac{\partial^{2} V}{\partial x^{\alpha} \partial x^{\beta}} & \frac{\partial^{2} V}{\partial x^{\alpha} \partial \theta^{a}} \\
\frac{\partial^{2} V}{\partial \theta^{a} \partial x^{\alpha}} & \frac{\partial^{2} V}{\partial \theta^{a} \partial \theta^{b}}+\frac{\partial^{2} V_{\epsilon}}{\partial \theta^{a} \partial \theta^{b}}
\end{array}\right]
$$

and $D^{2} K$ denotes the second derivative of the kinetic energy $K$ with respect to $\left(\dot{x}^{\alpha}, \dot{\theta}\right)$. We already know that $D^{2} K\left(x_{e}, \theta_{e}, 0,0\right)$ is a positive definite matrix. By linear algebra and $(2.1)$, we can make $D^{2} \tilde{V}\left(x_{e}, \theta_{e}\right)$ positive definite by choosing $V_{\epsilon}$ such that its second derivative at $\left(x_{e}, \theta_{e}\right)$ is positive definite and the magnitudes of its eigenvalues are large. Thus, $\tilde{E}$ has a minimum at $\left(x_{e}, \theta_{e}, 0,0\right)$, so it can be used as a Lyapunov function. We introduce the following input term

$$
\tilde{u}_{a}=c_{a}^{b} g_{b c} \dot{\theta}^{c}
$$

where $c_{a}^{b}$ is a negative definite matrix with respect to the $g_{a b}$ metric. Then, we have

$$
\frac{d}{d t} \tilde{E}=c_{a}^{b} g_{b c} \dot{\theta}^{a} \dot{\theta}^{c} \leq 0
$$

Hence, $\left(x_{e}, \theta_{e}, 0,0\right)$ is still an equilibrium of the closedloop system and becomes Lyapunov stable.

To prove the asymptotic stability of the equilibrium, we use the LaSalle invariance principle. By (2.5) and the fact that $\tilde{E}$ has a minimum at $\left(x_{e}, \theta_{e}, 0,0\right)$, there exists a number $c \in \mathbb{R}$ such that the set $\Omega_{c}:=$ $\left\{z=\left(x^{\alpha}, \theta^{a}, \dot{x}^{\alpha}, \dot{\theta}^{a}\right) \in T Q \mid \tilde{E}(z) \leq c\right\}$ becomes a nonempty, compact and positively invariant set. Define $\mathcal{E}:=\left\{z=\left(x^{\alpha}, \theta^{a}, \dot{x}^{\alpha}, \dot{\theta}^{a}\right) \in \Omega_{c} \mid \frac{d}{d t} \tilde{E}(z)=0\right\}=\{z=$ $\left.\left(x^{\alpha}, \theta^{a}, \dot{x}^{\alpha}, \dot{\theta}^{a}\right) \in \Omega_{c} \mid \dot{\theta}^{a}=0\right\}$. Let $\mathcal{M}$ be the largest invariant subset of $\mathcal{E}$. Instead of directly looking into the dynamics on $\mathcal{M}$, we follow the approach given in van der Schaft [1986] and Jalnapurkar and Marsden $[1999,2000]$. Let $\mathbb{F} \tilde{L}: T Q \rightarrow T^{*} Q$ be the Legendre transform induced from the Lagrangian $\tilde{L}$ (see, e.g., Marsden and Ratiu [1999]). Since the Lagrangian $\tilde{L}$ is regular, we can define $\tilde{H}: T^{*} Q \rightarrow \mathbb{R}$ by $\tilde{H}=\tilde{E} \circ \mathbb{F} \tilde{L}^{-1}$. Define $G_{b}: T Q \rightarrow \mathbb{R}$ by $G_{b}\left(x^{\alpha}, \theta^{a}, \dot{x}^{\alpha}, \dot{\theta}^{a}\right)=\dot{\theta}^{b}$ and $F_{b}: T^{*} Q \rightarrow \mathbb{R}$ by $F_{b}=G_{b} \circ \mathbb{F} \tilde{L}^{-1}$. Let $(q(t), \dot{q}(t)) \in T Q$ be a trajectory of the closed-loop Lagrangian system with the force $\tilde{u}$. Then it is well known that the curve $(q(t), p(t)) \in T^{*} Q$ defined by

$$
(q(t), p(t))=\mathbb{F} \tilde{L}(q(t), \dot{q}(t))
$$

satisfies the following Hamiltonian equations:

$$
\begin{aligned}
\dot{q}^{i} & =\frac{\partial \tilde{H}}{\partial p_{i}} \\
\dot{p}_{i} & =-\frac{\partial \tilde{H}}{\partial q^{i}}+\tilde{u}_{i}
\end{aligned}
$$

where $\tilde{u}_{i}$ is short for $\tilde{u}_{i} \circ \mathbb{F} \tilde{L}^{-1}$. Notice that $\mathbb{F} \tilde{L}\left(x_{e}, \theta_{e}, 0,0\right)$ becomes an equilibrium of the system (2.6) and that $\Omega_{c}, \mathcal{E}$ and $\mathcal{M}$ are diffeomorphically mapped into $T^{*} Q$ via $\mathbb{F} \tilde{L}$, if necessary after shrinking $\Omega_{c}$ into the domain of $\mathbb{F} \tilde{L}$. Let $\{$,$\} be the Poisson$ bracket on $T^{*} Q$ induced from the standard symplectic form on $T^{*} Q$ (see Marsden and Ratiu [1999] for the definition). Consider the set of functions defined by

$$
\mathcal{C}=\operatorname{span}\left\{F_{b},\left\{\tilde{H}, F_{b}\right\},\left\{\tilde{H},\left\{\tilde{H}, F_{b}\right\}\right\}, \ldots\right\}
$$

$b=1, \ldots, \operatorname{dim} G$, where the span is over the collection of all linear combinations with real coefficients. Define the codistribution $\mathrm{d} \mathcal{C}=\operatorname{span}\{\mathrm{d} g \mid g \in \mathcal{C}\}$.

The equilibrium $\left(x_{e}, \theta_{e}, 0,0\right)$ is an isolated equilibrium by the Morse Lemma since every equilibrium is a critical point of the energy $\tilde{E}$ and $\left(x_{e}, \theta_{e}, 0,0\right)$ is a nondegenerate critical point. Theorem 2.2 in Jalnapurkar and Marsden [2000] implies that the only trajectory in $\mathcal{M}$ is the equilibrium itself when the dimension of $\mathrm{d} \mathcal{C}$ is $2 n$ in a neighborhood of $\mathbb{F} \tilde{L}\left(x_{e}, \theta_{e}, 0,0\right)$, where $n$ is the dimension of the configuration space $Q$. Now, consider a more general case where there is a subcodistribution of $\mathrm{d} \mathcal{C}$ whose locally constant dimension is $(2 n-1)$ around the equilibrium. The subcodistribution defines a one-dimensional (regular) submanifold of $T^{*} Q$, which contains the invariant set $\mathbb{F} \tilde{L}(\mathcal{M})$ as well as the equilibrium. Since the equilibrium is stable and isolated, the flow in the one-dimensional submanifold should converge to the equilibrium if necessary after shrinking the 
domain. Thus the (bi-)invariant set $\mathcal{M}$ is the equilibrium itself. By the LaSalle invariance principle, the equilibrium is asymptotically stable. We have proved the following theorem.

Theorem 2.1 If (2.1) holds, then $\left(x_{e}, \theta_{e}, 0,0\right)$ is Lyapunov stabilizable. If in addition, $\operatorname{dim} \mathrm{dC} \geq(2 n-1)$ in a neighborhood of $\mathbb{F} \tilde{L}\left(x_{e}, \theta_{e}, 0,0\right)$, where $n=\operatorname{dim} Q$, then $\left(x_{e}, \theta_{e}, 0,0\right)$ becomes an asymptotically stable equilibrium of the closed-loop system with the input u given by (2.2) and (2.4).

We can also do the above process on the Lagrangian side without explicitly using the symplectic structure on $T^{*} Q$. The set $\mathcal{C}$ corresponds, under the Legendre transformation, to the set $\mathcal{D}=\left\{(d / d t)^{k} G_{b} \mid k \in 0 \cup \mathbb{N}\right\}$ where we regard $(d / d t)^{k} G_{b}$ 's as functions defined on $T Q$ by using the given differential equations. Then, Theorem 2.1 still holds with $\mathrm{d} \mathcal{C}$ replaced by $\mathrm{d} \mathcal{D}$. The difference is a matter of computational convenience.

\section{Kinetic and Potential Shaping.}

In this section we prove an asymptotic stabilization theorem that is analogous to that in Bloch, Chang, Leonard and Marsden [1999] by combining the previous potential shaping technique with that of kinetic shaping; however, instead of assuming that the potential has the special form $V\left(x^{\alpha}, \theta^{a}\right)=V_{1}\left(x^{\alpha}\right)+V_{2}\left(\theta^{a}\right)$, we assume a more general form and make hypotheses appropriate to the bracketing methodology.

Bloch, Chang, Leonard and Marsden [1999] considered an equilibrium that is a maximum of the original potential energy in the $x^{\alpha}$ variables, i.e.,

$$
\frac{\partial^{2} V}{\partial x^{\alpha} x^{\beta}}\left(x_{e}, \theta_{e}\right)<0 .
$$

In (2.3) we cannot make $D^{2} \tilde{E}\left(x_{e}, \theta_{e}, 0,0\right)$ definite by any choice of the function $V_{\epsilon}: G \rightarrow \mathbb{R}$. Hence, under such circumstances, potential shaping alone cannot succeed. We use kinetic shaping and potential shaping to stabilize such an equilibrium.

We assume that the simplified matching assumptions SM-1 - SM-5 as defined in Bloch, Chang, Leonard and Marsden [1999] hold. That is, we assume that $g_{a b}$ is constant, $g_{\alpha a, \delta}=g_{\delta a, \alpha}$ and $V$ satisfies

$$
\frac{\partial^{2} V}{\partial x^{\alpha} \partial \theta^{a}} g^{a d} g_{\beta d}=\frac{\partial^{2} V}{\partial x^{\beta} \partial \theta^{a}} g^{a d} g_{\alpha d} .
$$

These imply that the one form $g^{a c} g_{\alpha c} \mathrm{~d} x^{\alpha}$ is locally exact; therefore, there is a function $h: U \rightarrow \mathfrak{g}$ for an open subset $U$ in $S=Q / G$ such that $h^{a}\left(x_{e}\right)=0$ and

$$
\frac{\partial h^{a}}{\partial x^{\alpha}}=\left(\frac{\rho-1}{\rho}-\frac{1}{\sigma}\right) g^{a c} g_{\alpha c} .
$$

Define a new coordinate chart for $Q$ by:

$$
\left(X^{\alpha}, Y^{a}\right)=\left(x^{\alpha}, \theta^{a}+h^{a}\left(x^{\alpha}\right)\right) .
$$

This coordinate change induces the following new local coordinates for $T Q$ :

$$
\begin{aligned}
& \left(X^{\alpha}, Y^{a}, \dot{X}^{\alpha}, \dot{Y}^{a}\right) \\
& \quad=\left(x^{\alpha}, \theta^{a}+h^{a}\left(x^{\alpha}\right), \dot{x}^{\alpha}, \dot{\theta}^{a}+\frac{\partial h^{a}}{\partial x^{\beta}} \dot{x}^{\beta}\right) .
\end{aligned}
$$

Notice that this change of coordinates leaves the equilibrium $\left(x_{e}, \theta_{e}, 0,0\right)$ fixed.

According to the method of controlled Lagrangians, the controlled Lagrangian $L_{\tau, \sigma, \rho, \epsilon}$ and the controlled energy $E_{\tau, \sigma, \rho, \epsilon}$ (i.e., the Lagrangian and the energy for the closed-loop system) are given as

$$
\begin{aligned}
& L_{\tau, \sigma, \rho, \epsilon}=\frac{1}{2}\left(g_{\alpha \beta}-\left(\frac{\rho-1}{\rho}-\frac{1}{\sigma}\right) g^{a b} g_{\alpha a} g_{\beta b}\right) \dot{X}^{\alpha} \dot{X}^{\beta} \\
& \quad+g_{\alpha a} \dot{X}^{\alpha} \dot{Y}^{a}+\frac{1}{2} \rho g_{a b} \dot{Y}^{a} \dot{Y}^{b}-V^{\prime}\left(X^{\alpha}, Y^{a}\right)
\end{aligned}
$$

and

$$
\begin{gathered}
E_{\tau, \sigma, \rho, \epsilon}=\frac{1}{2}\left(g_{\alpha \beta}-\left(\frac{\rho-1}{\rho}-\frac{1}{\sigma}\right) g^{a b} g_{\alpha a} g_{\beta b}\right) \dot{X}^{\alpha} \dot{X}^{\beta} \\
+g_{\alpha a} \dot{X}^{\alpha} \dot{Y}^{a}+\frac{1}{2} \rho g_{a b} \dot{Y}^{a} \dot{Y}^{b}+V^{\prime}\left(X^{\alpha}, Y^{a}\right),
\end{gathered}
$$

where

$$
\begin{gathered}
V^{\prime}\left(X^{\alpha}, Y^{a}\right)=V\left(X^{\alpha}, Y^{a}\right)+\int_{C} \frac{\partial V}{\partial Y^{a}} \frac{\partial h^{a}}{\partial X^{\alpha}} d X^{\alpha} \\
+\tilde{V}_{\epsilon}\left(Y^{a}\right) .
\end{gathered}
$$

$\tilde{V}_{\epsilon}$ is an arbitrary function with $Y_{e}$ as a critical point and $\sigma$ and $\rho$ are constant control gains. The curve $C$ is defined as follows. Fix $\left(X_{e}^{\alpha}\right) \in S$. For each $\left(X^{\alpha}, Y^{a}\right) \in$ $S \times G$, we choose any curve $C \in S \times\left\{\left(Y^{a}\right)\right\}$ joining $\left(X_{e}^{\alpha}, Y^{a}\right)$ and $\left(X^{\alpha}, Y^{a}\right)$. We can check that $\left(X_{e}, Y_{e}\right)$ becomes a critical point of $V^{\prime}$. Notice that we have slightly abused notation in (3.6). $V\left(X^{\alpha}, Y^{a}\right)$ denotes the originally given potential energy $V$ expressed in the new coordinates.

If we define the control law to be

$$
u_{a}=-\frac{d}{d t}\left(\kappa g_{\alpha a} \dot{x}^{\alpha}\right)+\frac{\rho-1}{\rho} \frac{\partial V}{\partial \theta^{a}}-\frac{1}{\rho} \frac{\partial V_{\epsilon}}{\partial \theta^{a}}+\frac{1}{\rho} u_{a}^{\text {diss }}
$$

where $V_{\epsilon}=V^{\prime}-V$ and $u^{\text {diss }}$ is the dissipative part of the control law to be determined, then in the new coordinates, the Euler-Lagrange equations for the controlled system are

$$
\begin{aligned}
& \frac{d}{d t} \frac{\partial L_{\tau, \sigma, \rho, \epsilon}}{\partial \dot{X}^{\alpha}}-\frac{\partial L_{\tau, \sigma, \rho, \epsilon}}{\partial X^{\alpha}}=0, \\
& \frac{d}{d t} \frac{\partial L_{\tau, \sigma, \rho, \epsilon}}{\partial \dot{Y}^{a}}-\frac{\partial L_{\tau, \sigma, \rho, \epsilon}}{\partial Y^{a}}=u_{a}^{\text {diss }} .
\end{aligned}
$$

The expression for $u_{a}$ as a function of positions and velocities only is given in Bloch, Chang, Leonard and Marsden [1999]. 
Notice that $\left(x_{e}, \theta_{e}, 0,0\right)=\left(X_{e}, Y_{e}, 0,0\right) \in T Q$ is a critical point of $E_{\tau, \sigma, \rho, \epsilon}$ by choosing $\tilde{V}_{\epsilon}: G \rightarrow \mathbb{R}$ such that $Y_{e}$ is a critical point of $\tilde{V}_{\epsilon}$. The second derivative of $E_{\tau, \sigma, \rho, \epsilon}$ at $z_{e}=\left(X_{e}, Y_{e}, 0,0\right)$ is given by

$$
D^{2} E_{\tau, \sigma, \rho, \epsilon}\left(z_{e}\right)=\left.\left[\begin{array}{cc}
D^{2} V^{\prime} & 0 \\
0 & D^{2} K
\end{array}\right]\right|_{z=z_{e}}
$$

where

$$
\begin{aligned}
& D^{2} V^{\prime}\left(X_{e}, Y_{e}\right)= \\
& {\left[\begin{array}{lc}
\frac{\partial^{2} V}{\partial X^{\alpha} \partial X^{\beta}}+\frac{\partial V}{\partial Y^{a} \partial X^{\beta}} \frac{\partial h^{a}}{\partial X^{\alpha}} & \frac{\partial^{2} V}{\partial X^{\alpha} \partial Y^{a}}+\frac{\partial^{2} V}{\partial Y^{a} \partial Y^{b}} \frac{\partial h^{b}}{\partial X^{\alpha}} \\
\frac{\partial^{2} V}{\partial X^{\alpha} \partial Y^{a}}+\frac{\partial^{2} V}{\partial Y^{a} \partial Y^{b}} \frac{\partial h^{b}}{\partial X^{\alpha}} & \frac{\partial^{2} V}{\partial Y^{a} \partial Y^{b}}+\frac{\partial^{2} \tilde{V}_{\epsilon}}{\partial Y^{a} \partial Y^{b}}
\end{array}\right]}
\end{aligned}
$$

and $D^{2} K$ denotes the second derivative of the kinetic energy $K$ with respect to $\left(\dot{X}^{\alpha}, \dot{Y}^{a}\right)$. Notice that in (3.10) we used the fact that $\left(X_{e}, Y_{e}\right)$ is a critical point of $V$ and $Y_{e}$ is a critical point of $\tilde{V}_{\epsilon}$. The first block $\left(D^{2} V^{\prime}\right)_{11}\left(X_{e}, Y_{e}\right)$ of $D^{2} V^{\prime}\left(X_{e}, Y_{e}\right)$ can be expressed in the old coordinates as

$$
\begin{aligned}
& \left(D^{2} V^{\prime}\right)_{11}\left(X_{e}, Y_{e}\right)= \\
& \frac{\partial^{2} V}{\partial x^{\alpha} \partial x^{\beta}}-\left.\left(\frac{\rho-1}{\rho}-\frac{1}{\sigma}\right) \frac{\partial^{2} V}{\partial x^{\alpha} \partial \theta^{a}} g^{a c} g_{\beta c}\right|_{\left(x_{e}, \theta_{e}\right)} .
\end{aligned}
$$

Because of (3.1) we can find $\rho$ and $\sigma$ such that $\left(D^{2} V^{\prime}\right)_{11}\left(X_{e}, Y_{e}\right)$ is negative definite. Then, by simple linear algebra, we can find a function $\tilde{V}_{\epsilon}$ which has a maximum at $Y_{e}$ such that $D^{2} V^{\prime}\left(X_{e}, Y_{e}\right)$ becomes negative definite. Now, we consider the definiteness of $D^{2} K\left(X_{e}, Y_{e}, 0,0\right)$. As shown in Bloch, Chang, Leonard and Marsden [1999], $D^{2} K\left(X_{e}, Y_{e}, 0,0\right)$ becomes negative definite if SM-6 holds (i.e., the matrix $g_{a \alpha}\left(x_{e}^{\alpha}\right)$ is one-to-one), $\rho$ is negative and $\sigma$ satisfies

$$
1-\frac{1}{\sigma}>\max \lambda\left(x_{e}\right)
$$

where

$\lambda\left(x_{e}\right)=\left\{\lambda \mid \operatorname{det}\left(g_{\alpha \beta}\left(x_{e}\right)-\lambda g_{\alpha a}\left(x_{e}\right) g^{a b} g_{b \beta}\left(x_{e}\right)\right)=0\right\}$.

Hence, $D^{2} E_{\tau, \sigma, \rho, \epsilon}\left(X_{e}, Y_{e}, 0,0\right)$ becomes negative definite and thus $E_{\tau, \sigma, \rho, \epsilon}$ has a maximum at $\left(X_{e}, Y_{e}, 0,0\right)$. Accordingly, we can use $E_{\tau, \sigma, \rho, \epsilon}$ as a Lyapunov function.

Suppose we introduce the dissipative input

$$
u_{a}^{\mathrm{diss}}=c_{a}^{b} g_{b c} \dot{Y}^{c}
$$

where $c_{a}^{b}$ is a positive definite matrix in the $g_{a b}$ metric. Then, $\left(X_{e}, Y_{e}, 0,0\right)$ is still an equilibrium of the closedloop system. The time derivative of $E_{\tau, \sigma, \rho, \epsilon}$ is given by

$$
\frac{d}{d t} E_{\tau, \sigma, \rho, \epsilon}=c_{a}^{b} g_{b c} \dot{Y}^{b} \dot{Y}^{c} \geq 0
$$

which implies that $\left(X_{e}, Y_{e}, 0,0\right)$ is Lyapunov stable in the closed-loop system.

The next step is to prove asymptotic stabilization. Since $E_{\tau, \sigma, \rho, \epsilon}$ has a maximum at $\left(X_{e}, Y_{e}, 0,0\right)$ and it is non-decreasing, we can find $c \in \mathbb{R}$ such that the set $\Omega_{c}=\{z=$ $\left.\left(X^{\alpha}, Y^{a}, \dot{X}^{\alpha}, \dot{Y}^{a}\right) \in T Q \mid E_{\tau, \sigma, \rho, \epsilon}(z) \geq c\right\}$ is nonempty, compact and positively invariant. Define $\mathcal{E}=$ $\left\{z=\left(X^{\alpha}, Y^{a}, \dot{X}^{\alpha}, \dot{Y}^{a}\right) \in \Omega_{c} \mid \frac{d}{d t} E_{\tau, \sigma, \rho, \epsilon}(z)=0\right\}=$ $\left\{z=\left(X^{\alpha}, Y^{a}, \dot{X}^{\alpha}, \dot{Y}^{a}\right) \in \Omega_{c} \mid \dot{Y}^{a}=0\right\}$. Let $\mathcal{M}$ be the largest invariant subset of $\mathcal{E}$.

Let $\mathbb{F} L_{\tau, \sigma, \rho, \epsilon}: T Q \rightarrow T^{*} Q$ be the standard Legendre transform induced from the Lagrangian $L_{\tau, \sigma, \rho, \epsilon}$. Since the Lagrangian $L_{\tau, \sigma, \rho, \epsilon}$ is regular, we can define $H_{\tau, \sigma, \rho, \epsilon}: T^{*} Q \rightarrow \mathbb{R}$ by $H_{\tau, \sigma, \rho, \epsilon}=E_{\tau, \sigma, \rho, \epsilon} \circ \mathbb{F} L_{\tau, \sigma, \rho, \epsilon}^{-1}$. Define $G_{b}: T Q \rightarrow \mathbb{R}$ by $G_{b}\left(X^{\alpha}, Y^{a}, \dot{X}^{\alpha}, \dot{Y}^{a}\right)=\dot{Y}^{b}$. Let $F_{b}: T^{*} Q \rightarrow \mathbb{R}$ be defined by $F_{b}=G_{b} \circ \mathbb{F} L_{\tau, \sigma, \rho, \epsilon}^{-1}$. Consider the set of functions defined by

$\mathcal{C}=\operatorname{span}\left\{F_{b},\left\{H_{\tau, \sigma, \rho, \epsilon}, F_{b}\right\},\left\{H_{\tau, \sigma, \rho, \epsilon},\left\{H_{\tau, \sigma, \rho, \epsilon}, F_{b}\right\}\right\}, \ldots\right\}$,

where the span is over $b=1, \ldots, \operatorname{dim} G$, where $\{$,$\} is$ the standard Poisson bracket on $T^{*} Q$. Then, we can prove the following theorem.

Theorem 3.1 If SM-1 - SM-6 hold and there exist $\rho<$ 0 and $\sigma$ satisfying

$$
\frac{\partial^{2} V}{\partial x^{\alpha} \partial x^{\beta}}-\left.\left(\frac{\rho-1}{\rho}-\frac{1}{\sigma}\right) \frac{\partial^{2} V}{\partial x^{\alpha} \partial \theta^{a}} g^{a c} g_{\beta c}\right|_{\left(x_{e}, \theta_{e}\right)}<0
$$

and

$$
1-\frac{1}{\sigma}>\max \lambda\left(x_{e}\right)
$$

where

$\lambda\left(x_{e}\right)=\left\{\lambda \mid \operatorname{det}\left(g_{\alpha \beta}\left(x_{e}\right)-\lambda g_{\alpha a}\left(x_{e}\right) g^{a b} g_{b \beta}\left(x_{e}\right)\right)=0\right\}$,

then $\left(x_{e}, \theta_{e}, 0,0\right)$ is Lyapunov stabilizable. If in addition to the above assumptions, the dimension of the codistribution $\mathrm{d} \mathcal{C}$ is greater than or equal to $(2 n-1)$ in a neighborhood of $\mathbb{F} \tilde{L}\left(x_{e}, \theta_{e}, 0,0\right)$, where $n$ is the dimension of the configuration space $Q$, then $\left(x_{e}, \theta_{e}, 0,0\right)$ becomes an asymptotically stable equilibrium of the closed-loop system with the input u given by (3.7) and (3.13).

Generally, the bracket condition is computationally hard to check. Thus, sometimes, it is better to look into the dynamics on $\mathcal{M}$ case by case as we did in Bloch, Chang, Leonard and Marsden [1999].

Notice that (3.1) is not a part of the hypothesis of Proposition 3.1. Thus, using a similar argument, we can prove a variant of the above theorem:

Theorem 3.2 Assume that SM-1 - SM-5 hold, and that there exist $\rho>0$ and $\sigma$ such that $\left(g_{\alpha \beta}-\left(\frac{\rho-1}{\rho}-\frac{1}{\sigma}\right) g^{a b} g_{\alpha a} g_{\beta b}\right)\left(x_{e}\right)>0$ and

$$
\frac{\partial^{2} V}{\partial x^{\alpha} \partial x^{\beta}}-\left.\left(\frac{\rho-1}{\rho}-\frac{1}{\sigma}\right) \frac{\partial^{2} V}{\partial x^{\alpha} \partial \theta^{a}} g^{a c} g_{\beta c}\right|_{\left(x_{e}, \theta_{e}\right)}>0 .
$$


Then, $\left(x_{e}, \theta_{e}, 0,0\right)$ is Lyapunov stabilizable. If additionally the dimension of the codistribution $\mathrm{dC}$ is greater than or equal to $(2 n-1)$ in a neighborhood of $\mathbb{F} \tilde{L}\left(x_{e}, \theta_{e}, 0,0\right)$, where $n$ is the dimension of the configuration space $Q$, then there exists a feedback control law $u$ such that $\left(x_{e}, \theta_{e}, 0,0\right)$ becomes an asymptotically stable equilibrium of the closed-loop system.

\section{References}

Åström, K.J. and K. Furuta [1996] Swinging up a pendulum by energy control. IFAC, San Francisco 13.

Auckly, D., L. Kapitanski and W. White [1998] Control of nonlinear underactuated systems. Preprint.

Baillieul, J. [1993] Stable average motions of mechanical systems subject to periodic forcing. Fields Inst. Comm. 1, 1-23.

Bloch, A.M., D.E. Chang, N.E. Leonard and J.E. Marsden [1999] Controlled Lagrangians and the stabilization of mechanical systems II: Potential shaping and tracking. Preprint.

Bloch, A.M., D.E. Chang, N.E. Leonard, J.E. Marsden and C. Woolsey [2000] Asymptotic stabilization of Euler-Poincaré mechanical systems, Proc. IFAC Wkshp Lagrangian and Hamiltonian Methods for Nonlinear Control, Princeton, 56-61.

Bloch, A.M., P.S. Krishnaprasad, J.E. Marsden and G. Sánchez de Alvarez [1992] Stabilization of rigid body dynamics by internal and external torques. Automatica 28, 745-756.

Bloch, A.M., N.E. Leonard and J.E. Marsden [1997] Stabilization of mechanical systems using controlled Lagrangians, Proc CDC 36, 2356-2361.

Bloch, A.M., N.E. Leonard and J.E. Marsden [1998] Matching and stabilization by the method of controlled Lagrangians, Proc CDC 37, 1446-1451.

Bloch, A.M., N.E. Leonard and J.E. Marsden [1999a] Controlled Lagrangians and the stabilization of mechanical systems I: The first matching theorem, IEEE Trans. Aut. Control. To appear.

Bloch, A.M., N.E. Leonard and J.E. Marsden [1999b] Stabilization of the pendulum on a rotor arm by the method of controlled Lagrangians, Proc. of ICRA '99, IEEE, 500-505.

Bloch, A.M., N.E. Leonard and J.E. Marsden [1999c] Potential shaping and the method of controlled Lagrangians Proc CDC 38, 1652-1657.
Bloch, A.M., J.E. Marsden, G. Sánchez de Alvarez [1997] Stabilization of relative equilibria of mechanical systems with symmetry. Proc. Notre Dame Conf. on Applied Mathematics, Birkhäuser.

Hamberg, J. [1999] General matching conditions in the theory of controlled Lagrangians, Proc. $C D C, \mathbf{3 8}$, $2519-2523$.

Jalnapurkar, S.M. and J.E. Marsden [1999] Stabilization of relative equilibria II, Reg. and Chaotic Dyn. 3, 161-179.

Jalnapurkar, S.M. and J.E. Marsden [2000] Stabilization of relative equilibria, IEEE Trans. Auto. Control, to appear.

Koditschek, D.E. and E. Rimon [1990] Robot navigation functions on manifolds with boundary. Adv. in Appl. Math. 11, 412-442.

Krishnaprasad, P.S. [1985] Lie-Poisson structures, dual-spin spacecraft and asymptotic stability, Nonl. Anal. Th. Meth. and Appl. 9, 1011-1035.

Leonard, N.E. [1997] Stabilization of underwater vehicle dynamics with symmetry-breaking potentials, Systems and Control Letters 32, 35-42.

Marsden, J.E. [1992], Lectures on Mechanics London Mathematical Society Lecture note series. 174, Cambridge University Press.

Marsden, J.E. and T.S. Ratiu [1999] Introduction to Mechanics and Symmetry. Texts in Applied Mathematics, 17, Springer-Verlag, 1994. Second Edition, 1999.

Spong, M.W. [1996] Energy based control of a class of underactuated mechanical systems. IFAC, San Francisco 13, 431-436.

van der Schaft, A. J. [1986], Stabilization of Hamiltonian systems, Nonlinear Analysis, Theory, Methods and Applications, 10, 1021-1035.

Wang, L.S. and P.S. Krishnaprasad [1992] Gyroscopic control and stabilization, J. Nonlinear Sci. 2, $367-415$. 